\title{
Arachnoid Cyst in the Middle Cranial Fossa Presenting with Pulsatile Exophthalmos: Case Report and Literature Review
}

\author{
Atsushi SAITO, ${ }^{1}$ Hiroyuki KON, ${ }^{1}$ Shinya HARYU, ${ }^{1}$ Masaki MinO, ${ }^{1}$ \\ Tatsuya SASAKI, ${ }^{1}$ and Michiharu NISHIJIMA ${ }^{1}$
}

${ }^{1}$ Department of Neurosurgery, Aomori Prefectural Central Hospital, Aomori, Aomori

\begin{abstract}
A 20-year-old woman suffered gradual progression of right pulsatile exophthalmos and slight headache. Computed tomography (CT) demonstrated outward and downward displacement of the right globe and an arachnoid cyst in the right middle cranial fossa associated with thinned and anterior protrusion of a bony orbit. Microscopic cystocisternotomy was performed and the cerebrospinal fluid (CSF) inside of the cyst communicated into the carotid cistern and cistern in the posterior cranial fossa. Pulsatile exophthalmos improved immediately after surgery. Arachnoid cyst in the middle cranial fossa presenting with exophthalmos is rare. Microscopic cystocisternotomy might successfully improve CSF flow and relieve exophthalmos.
\end{abstract}

Key words: arachnoid cyst, exophthalmos, bone erosion, venous hypertension

\section{Introduction}

Arachnoid cysts are developmental cysts in origin, which contains a cerebrospinal fluid (CSF)-like fluid and are located usually within the arachnoidal membrane. ${ }^{1-3)}$ In the majority of cases, these cysts are asymptomatic, but in a small group of patients, they are symptomatic mainly because many of them expand slowly and displace the neighboring structures. ${ }^{3)}$ Arachnoid cyst can cause are modeling process of the bony wall due to chronically low pressure. ${ }^{3)}$ We experienced a rare case of arachnoid cyst in the middle cranial fossa presenting with pulsatile exophthalmos. Cystocisternostomy improved the symptom, suggesting that not only bony compression against the orbit, but also CSF circulatory disturbance might be responsible for the clinical manifestation. We report the rare clinical features and present a review of the literature.

\section{Case Report}

This 20-year-old woman found right exophthalmos 3 years before and suffered gradual progression of exophthalmos and slight headache. Symptoms aggravated gradually and she visited our department. She was alert

Received January 22, 2013; Accepted May 20, 2013 with no neurological deficit, including disturbance of visual acuity and the visual field. She complained of right pulsatile exophthalmos and retroocular pain. She had no significant past medical history and no head or facial trauma. Physical examination revealed no marked limitation of ocular and eyelid movement. Her telexophthalmometry showed $19 \mathrm{~mm}$ oculus dexter (OD) and $14 \mathrm{~mm}$ oculus sinister (OS). Visual acuity was 2.0 OD, 1.2 OS and intraocular pressure was $13 \mathrm{mmHg}$ OD and $12 \mathrm{mmHg}$ OS. No abnormal finding in bilateral fundus and optic media. Computed tomography (CT) demonstrated outward and downward displacement of the right globe and low density fluid collection in the right middle cranial fossa, suggesting arachnoid cyst (Fig. 1). The cyst had thinned the anteromedial wall of the middle cranial fossa with a compressive sign of bony swallowing fossa against the orbital space on bone CT images (Fig. 1). Magnetic resonance images (MRIs) showed CSFlike fluid collection in the cystic lesion and compressive findings against the posterior region of the orbit (Fig. 2). Pre-operative phase-contrast cine MRI showed that cystic fluid wobbled fast and did not synchronize with ventricular and cisternal CSF movement. Microscopic cystocisternostomy was performed. Right frontotemporal osteoplastic craniotomy was performed and sylvian fissure was opened. Middle cranial fossa, lateral side of posterior orbital wall, and carotid cistern were exposed. Cyst wall 

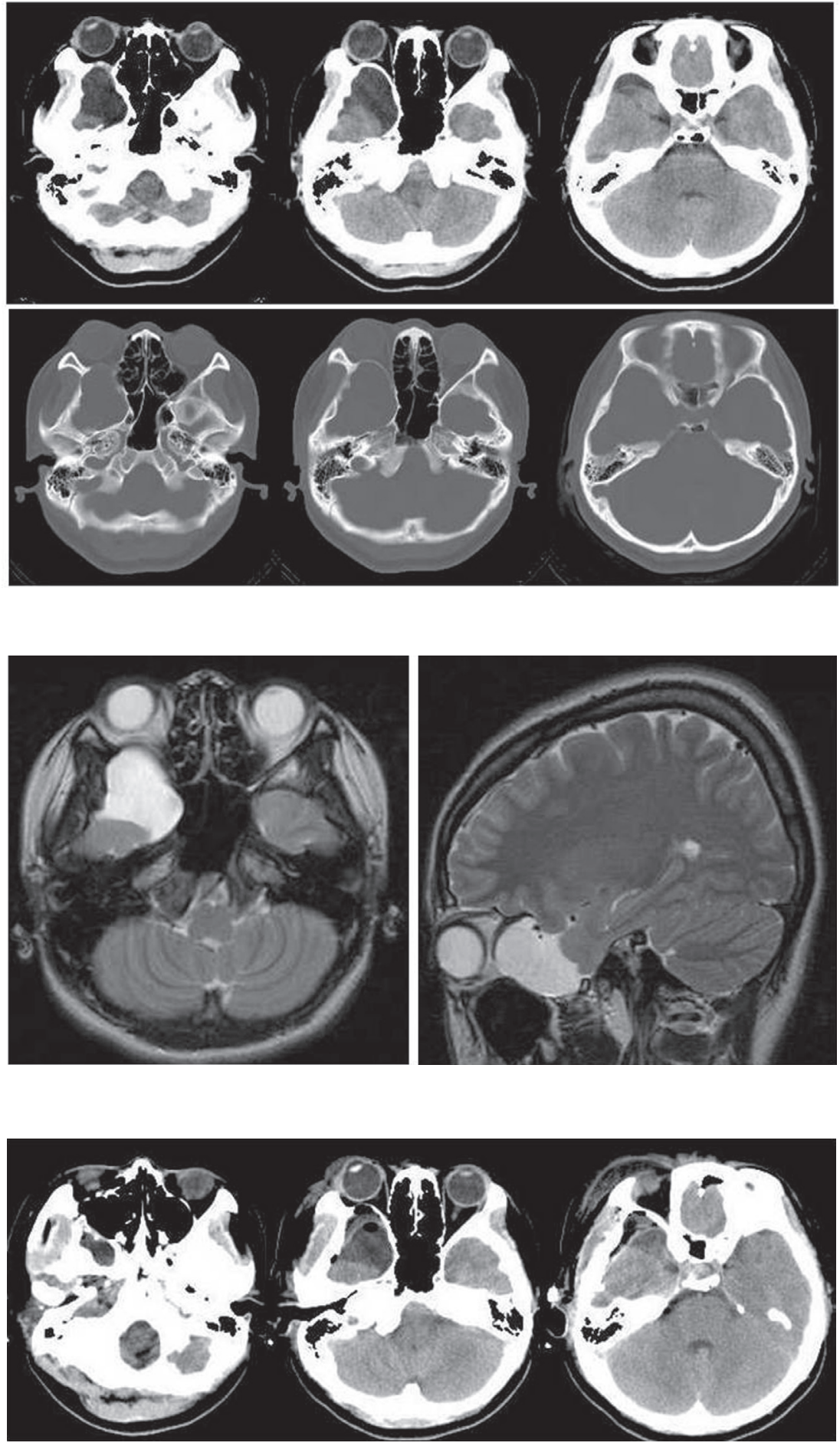

Fig. 1 Computed tomography (CT) images showed a cystic lesion containing low density, cerebrospinal fluid (CSF)-like contents in the right anterior middle cranial fossa, suggesting arachnoid cyst (upper). Bone CT images revealed an association with a bony deformity of the posterior orbital wall, which was thinned and anteriorly swollen (lower).

Fig. 2 Magnetic resonance images showed cerebrospinal fluid (CSF)-like fluid collection in the cystic lesion and compressive findings against the posterior region of the orbit (upper: $\mathrm{T}_{2}$-weighted axial image, lower: $\mathrm{T}_{2}$-weighted sagittal image).

Fig. 3 Postoperative computed tomography images showed decompression and shrinkage of the arachnoid cyst in the right middle cranial fossa. and the Liliequist membrane were incised and CSF inside the cyst became clear (Fig. 3). Posterior wall of orbit was thin and partial bony defect was observed via the middle fossa dura mater. CSF inside the cyst communicated into the carotid cistern and cistern in the posterior cranial fossa (Fig. 3). Pathological examination showed a monolayer cystic wall composed of squamous epithelium. Postoperative CT showed decompression of the arachnoid cyst in the middle cranial fossa. Post-operative finding on phase-contrast cine MRI showed that wobbling of cystic fluid changed slower and larger and synchronized with ventricular and cisternal CSF movement compared with that on pre-operative cine MRI. Pulsatile exophthalmos improved immediately after surgery. No re-expansion of the cyst or exophthalmos has been observed 5 years after surgery. 


\section{Discussion}

Arachnoid cysts are benign lesions lying entirely within the arachnoid membrane. ${ }^{3-5)}$ The membrane of the cyst is very thin, and the inner side is covered by flattened arachnoid cells, which secretes a colorless fluid like CSF although, occasionally, xanthochromic, proteinaceous, or hemorrhagic fluid is identified. ${ }^{3)}$ Large arachnoid cysts are thought to represent mainly cysts isolated from the subarachnoid space, and these cysts can be increased in size and they compress the underlying cerebral parenchyma or other structures. ${ }^{6}$ )

Expansion of the arachnoid cyst, or at least failure of the cyst to collapse, suggests a mechanism that maintains or increases its fluid volume. ${ }^{7)}$ The remodeling process is a well-known phenomenon. Tsitouridis et al. reported that bone tissue undergoes continual renewal with osteoclasts, which resorbs the calcified matrix and osteoblasts, and synthesize a new bone matrix. ${ }^{3)}$ In the presence of chronic pressure upon the bone, osteoclasts resorb the bone matrix but, simultaneously on the other side, osteoblasts synthesize new bones. ${ }^{8,9)}$ The timing for surgical decompression is dependent mainly on the degree of the exophthalmos, the location of the cyst, and the age of the patient. ${ }^{10,11)}$

Tsitouridis et al. reported 23 cases of arachnoid cysts in the frontal part of the temporal bone and discussed the radiological and clinical features. ${ }^{3)}$ All the cases had clinical exophthalmos and the periodic appearance of headache, and five had seizures. They categorized the exophthalmos into three groups: mild, moderate, and large, and categorized the size as: small, medium, and large. The majority had mild exophthalmos in 16/23 and small arachnoid cyst in 13/23. The peak point of bowing of the posterior wall of the orbit was in the posterior third in 20/23. Fisher et al. reported two cases of arachnoid cyst that altered the bony orbit. ${ }^{2}$ Two cases had proptosis and disturbance of ocular movement caused by arachnoid cysts in the middle cranial fossa. Radiological and ophthalmological findings did not change for 5 years. In the other case, diplopia aggravated for 2 years after the initial diagnosis. Medial rectus recession was performed and the symptom improved. There are few reports about the surgical therapeutic strategy for progressively symptomatic cases and surgical outcomes.

In our case, chief complaints were pulsatile exophthalmos, and repeated slight headache. These symptoms were immediately relieved after cystocisternostomy in spite of the postoperative finding that orbital bony deformity remained. This is the first report that cystocisternostomy successfully improved pulsatile exophthalmos in middle cranial fossa arachnoid cyst to our knowledge. The orbital posterior wall became thin and anteriorly swollen, and bony compression against the orbital space was suspected to be responsible; however, improvement of CSF circulation due to cystocisternostomy might be the dominant cause of pulsatile exophthalmos. Enlargement of arachnoid cyst in the middle cranial fossa might contribute to remodeling of the bony structure and result in deformity of orbital posterior wall. There is a possibility that the water-hammer effect due to disturbance of focal CSF circulation inside of the cyst might cause not only bony remodeling, but also direct bulbar compression. Intraoperative findings showed partial bone defect of orbital posterior wall and CSF pulsatile pressure might travel intraorbital structures via dura mater uncovered with bony structures. Postoperative cine-MRI showed that wobbling of cystic fluid changed slower and synchronized with ventricular and cisternal CSF movement. There is a possibility that cystocisternostomy might improve limited intracystic CSF circulation and relieve CSF pulsatile pressure. Incision of the cyst wall and connection to the cistern should relieve expansive wall pressure and decrease the water-hammer effect of the arachnoid cyst. No recurrence of arachnoid cyst expansion was observed, but bony deformity remained on CT 5 years after surgery. A long-term follow-up is required to evaluate the remodeling process under the condition without the water-hammer effect of the cyst.

The following preoperative findings were not completely evaluated and there are limitations in clinical and radiological assessments: (1) Onset of pulsatile exophthalmos is unclear. The patient did not have past history of sudden aggravation and head injury. We could not evaluate radiological findings before and after pulsatile exophthalmos. However, intraoperative finding of partial bony defect of posterior orbital wall indicates that gradual progression of bony thinning and defect might expand exposure of dural surface and worsen pulsatile compression into orbit resulting in symptoms. (2) Existence of disturbance of focal CSF circulation is unclear. We could evaluate dynamic state of intracystic fluid with only cine-MRI preoperatively. Radiological findings of cine-MRI and comparison between before and after cystocisternostomy are not quantitative data and not enough to be objectively evaluated. However, slower and synchronized movement with intraventricular or cisternal CSF of cystic fluid in post-cystocisternostomy indicates a possibility of enlargement of cystic fluid circulation area and dispersion of intracystic pulsatile pressure. These findings indicate the existence of pre-operative disturbance of focal CSF circulation. Detail assessments of communication of inside and outside of cystic fluid, existence of one way-check valve structure of cystic wall, and bony defect of orbital wall should have been evaluated preoperatively. (3) Surgical indication of pulsatile exophthalmos is unclear. Cosmetic improvement for orbit deformity due to exophthalmos needs plastic surgery. However, treatment of pulsatile exophthalmos needs reduction of intracystic pulsatile pressure or repairment of bony defect of orbital wall.

We experienced a rare case of arachnoid cyst in the 
middle cranial fossa presenting with pulsatile exophthalmos. Cystocisternotomy improved the symptom, suggesting that not only bony compression against the orbit, but also CSF circulatory disturbance might be responsible for the clinical manifestation even in an expansive arachnoid cyst associated with bony compression.

\section{Conflicts of Interest Disclosure}

The authors report no conflicts of interest concerning the case report or the findings specified in this article.

\section{References}

1) Duz B, Kaya S, Daneyemez M, Gonul E: Surgical management strategies of intracranial arachnoid cysts: a single institution experience of 75 cases. Turk Neurosurg 22: 591-598, 2012

2) Fisher T, Nugent R, Rootman J: Arachnoid cysts with orbital bone remodeling-two interesting cases. Orbit 24: 59-62, 2005

3) Tsitouridis I, Papastergiou C, Emmanouilidou M, Goutsaridou F, Chondromatidou S, Eleni T: Arachnoid cyst of the frontal part of the temporal lobe producing exopthtalmos CT and MRI evaluation. Clin Imaging 26: 302-305, 2002
4) Robinson RG: Congenital cysts of the brain: arachnoid malformations. Prog Neurol Surg 1: 133-174, 1971

5) Smith RA, Smith WA: Arachnoid cysts of the middle cranial fossa. Surg Neurol 5: 246-252, 1976

6) Flodmark O: Neuroradiology of selected disorders of the meninges, calvarium, and venous sinuses. AJNR Am J Neuroradiol 13: 483-491, 1992

7) Go KG, Hew JM, Kamman RL, Molenaar WM, Pruim J, Blaauw EH: Cystic lesions of the brain. A classification based on pathogenesis, with consideration of histological and radiological features. Eur J Radiol 17: 69-84, 1993

8) Hill PA: Bone remodeling. Br J Orthod 25: 101-107, 1998

9) Laugier P, Berger G, de Vernejoul MC: [Bone remodeling: evaluation methods]. Rev Prat 48: 1185-1190, 1998 (French)

10) Jallo GI, Woo HH, Meshki C, Epstein FJ, Wisoff JH: Arachnoid cysts of the cerebellopontine angle: diagnosis and surgery. Neurosurgery 40: 31-37; discussion 37-38, 1997

11) Raimondi AJ, Shimoji T, Gutierrez FA: Suprasellar cysts: surgical treatment and results. Child's Brain 7: 57-72, 1980

Address reprint requests to: Atsushi Saito, MD, PhD, Department of Neurosurgery, Aomori Prefectural Central Hospital, 2-1-1 Higashi Tsukurimichi, Aomori, Aomori 030-0910, Japan. e-mail: satsushi2002@yahoo.co.jp 